\title{
Performance analysis of photonic RF self-interference cancellation for full- duplex communication
}

Su, Xinxin, Fu, Shuanglin, Wang, Shuo, Yang, Xu, Wang, Pengyi, et al.

Xinxin Su, Shuanglin Fu, Shuo Wang, Xu Yang, Pengyi Wang, Chao Wang, Zhenlin Wu, Yiying Gu, Mingshan Zhao, Xiuyou Han, "Performance analysis of photonic RF self-interference cancellation for full-duplex communication," Proc. SPIE 11192, Real-time Photonic Measurements, Data Management, and Processing IV, 111920H (20 November 2019); doi: 10.1117/12.2538562

SPIE. Event: SPIE/COS Photonics Asia, 2019, Hangzhou, China 


\title{
Performance Analysis of Photonic RF Self-Interference Cancellation for Full-Duplex Communication
}

\author{
Xinxin $\mathrm{Su}^{\mathrm{a}}$, Shuanglin $\mathrm{Fu}^{\mathrm{a}}$, Shuo Wang ${ }^{\mathrm{a}}$, Xu Yang ${ }^{\mathrm{b}}$, Pengyi Wang ${ }^{\mathrm{b}}$, Chao Wang ${ }^{\mathrm{c}}$, Zhenlin $\mathrm{Wu}^{\mathrm{a}}$,Yiying \\ $\mathrm{Gu}^{\mathrm{a}}$ and Mingshan Zhao ${ }^{\mathrm{a}}$, Xiuyou Han ${ }^{\mathrm{a},{ }^{*}}$ \\ ${ }^{a}$ School of Optoelectronic Engineering and Instrumentation Science, Dalian University of \\ Technology, Dalian, 116024, China; \\ ${ }^{b}$ CETC Key Laboratory of Aerospace Information Applications, Shijiazhuang, 050081, China; \\ ${ }^{\mathrm{c}}$ School of Engineering and Digital Arts University of Kent Canterbury, CT2 7NT, U.K. \\ *Corresponding author, e-mail: xyhan@dlut.edu.cn
}

\begin{abstract}
A photonic RF self-interference cancellation (SIC) scheme for full-duplex communication is proposed and demonstrated experimentally. It is based on phase modulation to convert the RF signal into optical domain. The interference cancellation performance of the photonic RF SIC system under different delay deviation $(\Delta \tau)$ and amplitude deviation $(\Delta \alpha)$ is analyzed. The cancellation depth of $34.5 \mathrm{~dB}$ is measured for $10 \mathrm{GHz}$ signal with bandwidth of $50 \mathrm{MHz}$. According to experimental results, the interference cancellation performance affected by the time delay deviation, the amplitude deviation and the phase response is investigated. The results give a direction for the improvement of system performance.
\end{abstract}

Keywords: full-duplex communication, RF self-interference cancellation, phase modulation, optical sideband filtering

\section{INTRODUCTION}

In the field of wireless communications, compared to Time Division Duplex (TDD) and Frequency Division Duplex (FDD), Full-Duplex can realize simultaneous transmitting and receiving signals at the same frequency, which increases spectrum utilization significantly and doubles data rate [1-3]. However, during the full-duplex communication, the transmitted signal due to the arrangement of the antenna or the like may cause interference in the same frequency of the received useful signal, which is called RF self-interference or co-location interference, and cannot be filtered by a notch filter or a narrow passband filter [1]. Therefore, RF self-interference cancellation (SIC) is very urgent for the application of Full-Duplex. In recent years, the electronic RF self-interference cancellation methods for full-duplex communication have been widely investigated [4], [5]. However, this kind of method suffers from narrow bandwidth, low time delay precision and vulnerable to electromagnetic interference.

Compared with electronic methods, photonic RF self-interference cancellation approaches with the advantages of wider operational bandwidth, higher time delay precision, and immunity to electromagnetic interference are being attracted more and more attentions [6]-[14]. In [6] two Mach-Zehnder modulators (MZMs) biased at inverted quadrature points are utilized to implement the electrical-to-optical $(\mathrm{E} / \mathrm{O})$ conversion of all the received signals, including the weak signal of interest (SOI) and the strong interference signal, and the tapped reference signal from the transmitter. After tuning the delay time and amplitude of the received signals in the optical domain by a tunable optical delay line (TODL) and a variable optical attenuator (VOA), the interference and reference signals cancel each other out upon a photo detector due to exact out of phase and however identical magnitude, and the SOI was recovered. In [7], an analog optical self-interference cancellation system for over-the-air multipath cancellation was demonstrated. Channel matching was performed entirely in the optical domain with VOA and TODL. A single mode to multimode combiner enables the cancellation of up to six multipath components using just two optical sources. This program reflects the actual situation and over $35 \mathrm{~dB}$ of cancellation is achieved. A scheme based on two polarization modulators (PolMs) and a dispersive element was proposed in [9] for analog multipath interference cancellation. Multiple optical compensation branches are formed by an array of tunable lasers. In [10] an integrated dual-parallel MZM (DP-MZM) is proposed for RF selfinterference cancellation with the sub-MZM in the lower path operating at the minimum bias point and resulting in the double sideband with carrier-suppressed (DSB-CS) signal. Due to the optical carrier being suppressed in the lower path the optical interference at the $\mathrm{Y}$ branch waveguide is avoided and signal of interest can be recovered with a high gain. In [13] and [14] the integrated microwave photonic circuit for active, analog self-interference cancellation is designed and

Real-time Photonic Measurements, Data Management, and Processing IV, edited by Ming Li,

Bahram Jalali, Mohammad Hossein Asghari, Proc. of SPIE Vol. 11192, 111920H

(C) 2019 SPIE · CCC code: 0277-786X/19/\$21 - doi: 10.1117/12.2538562 
demonstrated experimentally. The cascaded semiconductor optical amplifiers (SOAs) simultaneously control the amplitude and phase, showing a compact sub-system and promising low power consumption.

In this paper, we present a photonic RF SIC scheme for full-duplex communication by using phase modulation and optical sideband filtering. Without the complicated bias voltage control as needed in conventional intensity modulation schemes [6], [10], the system is greatly simplified. The theoretical model of the photonic RF SIC system is established. The factors influencing the RF SIC performance including the time delay, the amplitude deviation and the phase deviation are investigated with simulation and experimental measurement.

\section{SYSTEM STRUCTURE AND PERFORMANCE ANALYSIS}

The schematic of the proposed optical RF SIC system is shown in Figure 1. Two lasers with different wavelengths of $\lambda_{1}$ and $\lambda_{2}$ are sent to two phase modulators $\mathrm{PM}_{1}$ and $\mathrm{PM}_{2}$, respectively. The received RF signal $s(t)+i(t)$ from the receiver antenna, where $s(t)$ is signal of interest and $i(t)$ is interference signal, is modulated on the light wave of $\lambda_{1}$ via $\mathrm{PM}_{1}$. The tapped RF signal $r(\mathrm{t})$ from the transmitter (Tx), as the reference signal, is modulated on the light wave of $\lambda_{2}$ via $\mathrm{PM}_{2}$. The insets (A) and (B) in Figure 1 show the spectra of the phase-modulated optical signals output from $\mathrm{PM}_{1}$ and $\mathrm{PM}_{2}$. It can be seen that the sidebands of the phase-modulated optical signals have the inherent out of phase relationship, namely, there is a $\pi$ phase difference between the right sideband and the left sideband. Through a VOA and a TODL, the phasemodulated signal in the lower path is combined with the phase-modulated signal in the upper path are fed to an optical filter (OF). The left sidebands of the phase-modulated optical signals in the upper path and the right sideband of the phase-modulated optical signal are filtered out, generating the singles of the single sideband signal with carrier (SSB $+\mathrm{C}$ ), as shown in the inset $(C)$ in Figure 1. After being filtered by the OF, the optical signals are input to the photodetector (PD), upon which the electrical signals are converted and the interference signal is cancelled by the reference signal.

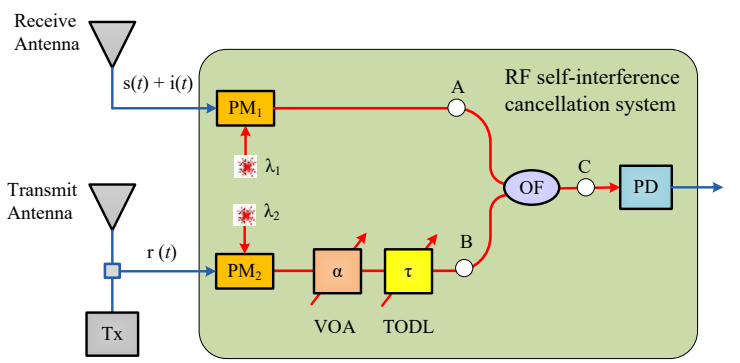

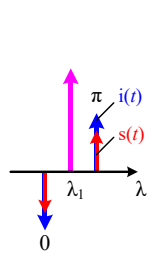

(A)

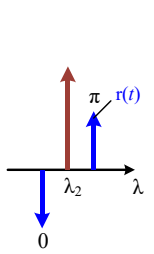

(B)

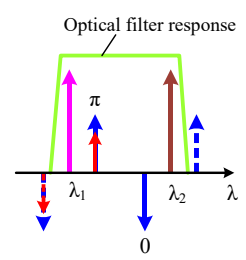

(C)

Figure 1. The schematic of the proposed optical RF SIC system. PM, phase modulator; VOA, variable optical attenuator; TODL, tunable optical delay line; OF, optical filter; PD, photodetector; Tx, transmitter. The insets show the spectra of the phase-modulated optical signals before (A and B) and after $(\mathrm{C})$ the $\mathrm{OF}$.

The system cancellation depth is investigated by the average power ratio of the interference signal and reference signal, which is defined as

$$
S R=\frac{\frac{1}{T} \int_{0}^{T}\left|i_{1}+i_{2}\right|^{2} d t}{\frac{1}{T} \int_{0}^{T} i_{1}^{2} d t}
$$

where $i_{1}$ and $i_{2}$ are the current of interference signal and reference signal output from the photonic RF SIC system, respectively.

According to the Pascal theorem, the energy value of the signal with a certain bandwidth in the time domain can be expressed as

$$
S R(x, \Delta \tau, B)=10 \lg \left[1+(1-x)^{2}-2(1-x) \sin c(B \Delta \tau) \cos (2 \pi f \Delta \tau)\right]
$$

where $f$ is the signal frequency, $B$ is the signal bandwidth, $\Delta \tau$ is the time delay deviation of the two paths, and the amplitude mismatch is indicated as $x$, which is directly related to amplitude deviation $\Delta \alpha$. According to the theory above, the cancellation performance of the proposed photonic RF SIC system is analyzed as follows. 


\subsection{The time delay deviation}

The effects of time delay deviation on interference cancellation depth is investigated and a simulation is carried out. During the simulation, the amplitude is assumed as being matched, and the additional phase from the filter is not considered. When the delay deviation is large, such as in the range of $50 \mathrm{ps}$ to $1000 \mathrm{ps}$, the cancellation depth is changed periodically with different time delay deviation for a certain center frequency, and the period will be shorter as the center frequency being higher. In one period, when the delay deviation is within $1 \mathrm{ps}$, the cancellation depth is decreased with the delay deviation being increased. For the same delay deviation, the cancellation will be decreased when the center frequency is higher. Therefore, the smaller time delay deviation is needed for higher frequency to achieve a good cancellation depth.

In the practical system, the time delay deviation is determined by the adjustment accuracy of the optical delay line. The cancellation depth with different frequencies at different time delay accuracy is also simulated and the results are shown in Figure 2. It can be seen that cancellation depth is increased with the time delay accuracy being higher for a certain frequency. Therefore, the optical time delay line with higher accuracy is needed to achieve deeper cancellation depth.

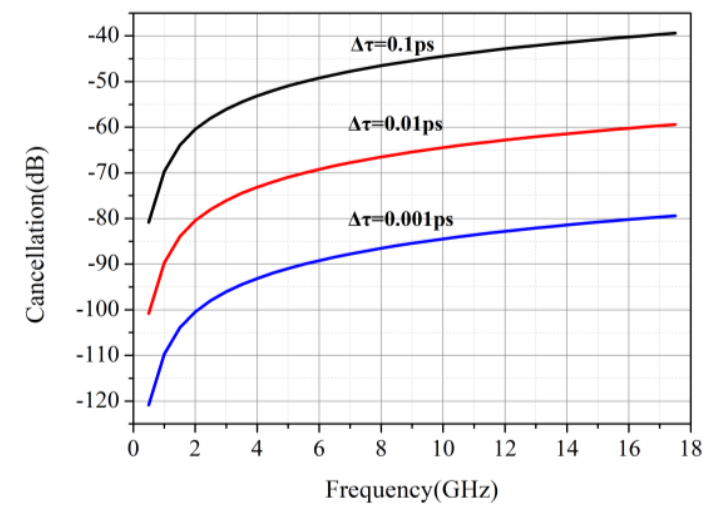

Figure 2. Cancellation depth with RF frequency at different delay accuracy.

\subsection{The amplitude deviation}

We analyze the cancellation depth under different amplitude deviation values when the delay deviation is $0.1 \mathrm{ps}$ and $0.01 \mathrm{ps}$, respectively, as shown in Figure 3. As the magnitude of the amplitude deviation increases, from $0.0001 \mathrm{~dB}$ to $1 \mathrm{~dB}$, the cancellation depth increases linearly. When the delay mismatch value is changed from $0.1 \mathrm{ps}$ to $0.01 \mathrm{ps}$, the variation of the cancellation depth becomes larger with the fixed amplitude deviation value. It is much obvious when the amplitude deviation value is less than $0.01 \mathrm{~dB}$. Moreover, when the delay and attenuation mismatch values are constant, the cancellation depth will deteriorate as the frequency increases. Therefore, in order to achieve a desired cancellation depth, the higher frequency, the higher accuracy of the amplitude tuning is needed.

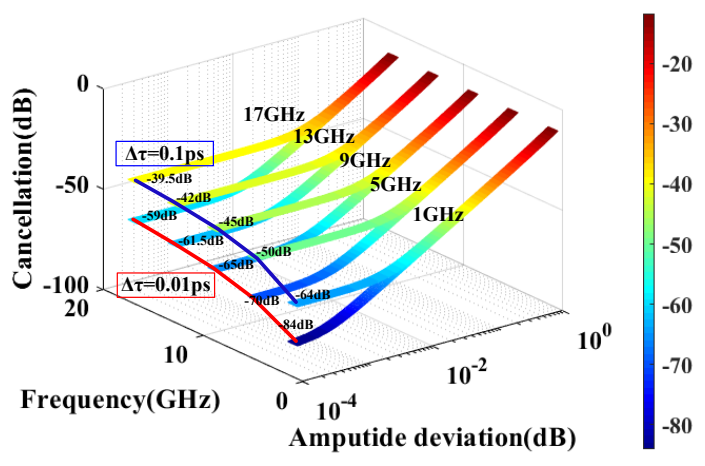

Figure 3. Cancellation depth with amplitude deviation at different frequencies (blue line indicates delay mismatch $=0.1 \mathrm{ps}$, red line indicates delay mismatch $=0.01 \mathrm{ps}$ ). 


\section{EXPERIMENT RESULTS AND DISCUSSION}

An experiment based on the setup shown in Figure 4 is performed, where the photonic RF self-interference cancellation system is the structure in Figure 1. The RF signal from a signal generator (SG1, Agilent E8257D) is used as the signal of interest $s(t)$. The RF signal from a second signal generator (SG2, Agilent E8267D) is split to two parts by an electronic $3 \mathrm{~dB}$ splitter. One part is input to $\mathrm{PM}_{2}\left(\mathrm{EO}\right.$ Space, $\mathrm{V}_{\pi}=4 \mathrm{~V}, 40 \mathrm{GHz}$ ) as the reference signal $r(t)$ and the other part is used as the interference signal $i(t)$. The RF signal $s(t)$ from SG1 and the RF signal $i(t)$ split from SG2 are combined as the received signals $s(t)+i(t)$ via an electronic $3 \mathrm{~dB}$ combiner, and are input to $\mathrm{PM}_{1}$ (EO Space, $\mathrm{V}_{\pi}=4 \mathrm{~V}, 40 \mathrm{GHz}$ ). A distributed feedback laser diode (DFB-LD, Emcore-1772) with a wavelength of $\lambda_{1}=1549.9 \mathrm{~nm}$ and a output power of 13 $\mathrm{dBm}$, and a tunable laser (NKT, DK-3460) with a wavelength of $\lambda_{2}=1549.5 \mathrm{~nm}$ and a output power of $13 \mathrm{dBm}$ are used as the light sources in the upper path and the lower path, respectively. Two phase modulators (PM1 and PM2) implement the E/O phase modulation of the received RF signal $s(t)+i(t)$ and the reference RF signal $r(t)$, respectively. After a VOA and a TODL, both of the phase-modulated optical signal in the lower path and the upper path were fed into the OF by which the left sideband of the received RF modulated optical signal and the right sideband of the reference RF modulated optical signal are filtered out. Therefore, the RF signals can be recovered by the $\mathrm{O} / \mathrm{E}$ conversion from the SSB + C optical signals upon the PD (Miteq SCMR-10M18G). The detected RF signals are measured with an electrical spectrum analyzer (ESA, Agilent E4440A). A vector network analyzers (Keysight Technologies N5225B) was used to measure the cancellation performance and the response of each channel.

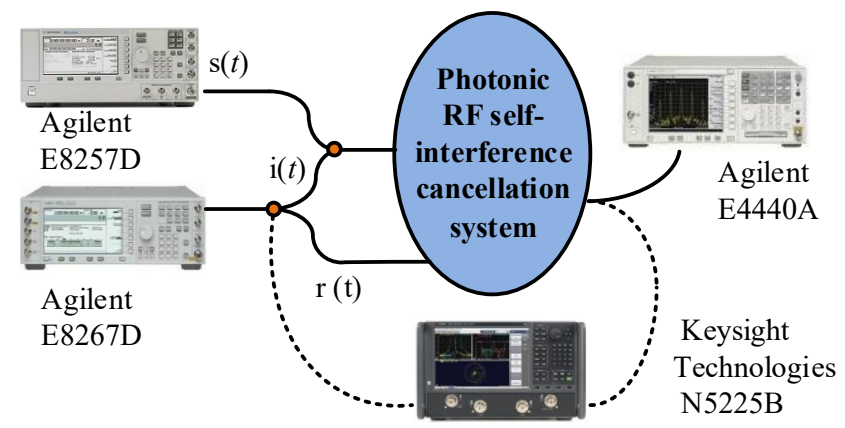

Figure 4. The setup for the performance measurement of the photonic RF SIC system.

In order to investigate the cancellation performance for RF signal with a certain bandwidth, the additive white Gaussian noise (AWGN) with a bandwidth of $50 \mathrm{MHz}$ and a power of $10 \mathrm{dBm}$ is modulated on the RF signal at $10 \mathrm{GHz}$, from SG2. The single tone signal from SG1 is used as the signal of interest. With the laser source of $\lambda_{2}$ being turned off, a strong interference signal is observed in the electrical spectrum as shown the blue curve in Figure 5. Then, by tuning on the laser source of $\lambda_{2}$, the $50 \mathrm{MHz}$ bandwidth interference signal is suppressed greatly with a cancellation depth as high as $34.5 \mathrm{~dB}$ and the desired $10 \mathrm{GHz}$ signal is maintained, as shown the red curve in Figure 5.

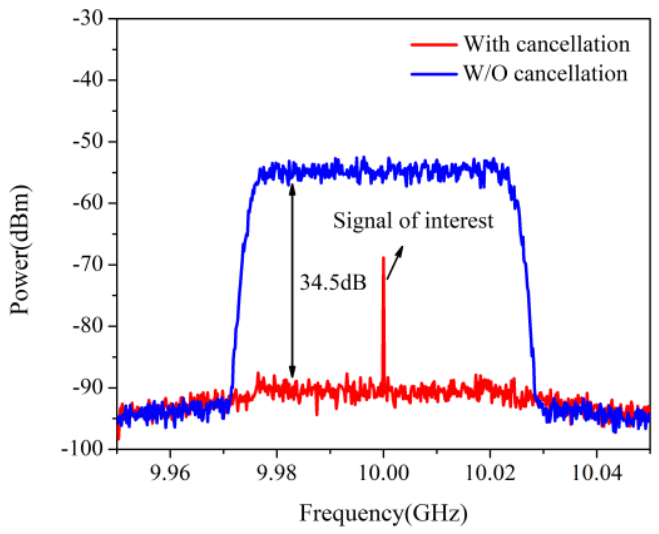

Figure 5. The measured RF spectra with and without SIC at bandwidth of $50 \mathrm{MHz}\left(f_{\mathrm{RF}}=10 \mathrm{GHz}\right)$. 
To further characterize the performance of the RF SIC system, a vector network analyzer is used to measure the cancellation depth of the system over a broad band with the results shown in Figure 6. It can be seen that the cancellation depth of $25.5 \mathrm{~dB}$ and $18.6 \mathrm{~dB}$ are obtained for the bandwidth of $100 \mathrm{MHz}$ and $300 \mathrm{MHz}$, respectively. The cancellation depth degrades with the bandwidth being increased. It is due to the more phase mismatch and amplitude mismatch over a wider bandwidth.

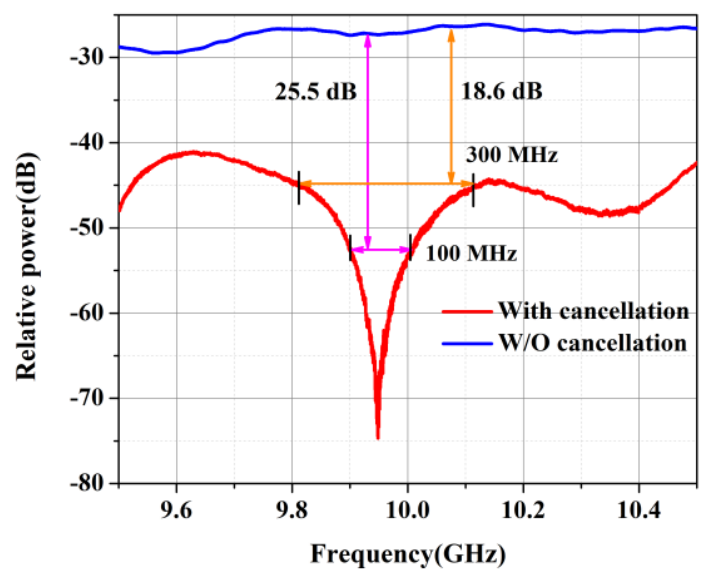

Figure 6. The measured RF spectra with and without RF SIC over a broad band.

Here we give a further analysis of the above experimental results. The phase response of the two channels is measured over $1 \mathrm{GHz}$ at the central frequency of $10 \mathrm{GHz}$ by the vector network analyzer. The phase difference between the two microwave photonic links is given as the blue curve in Figure 7, which floats up and down around 180 degree. It can be seen that out of phase relationship between the upper and lower paths is realized basically. The amplitude response difference of the upper and lower paths is shown the red curve in Figure 7. The cancellation depth over the bandwidth of $100 \mathrm{MHz}$ is higher than that over the bandwidth of $1 \mathrm{GHz}$, which is mainly caused by amplitude mismatch and phase mismatch. Therefore, the smaller phase and amplitude mismatch value over a certain bandwidth, the greater cancellation depth can be achieved.

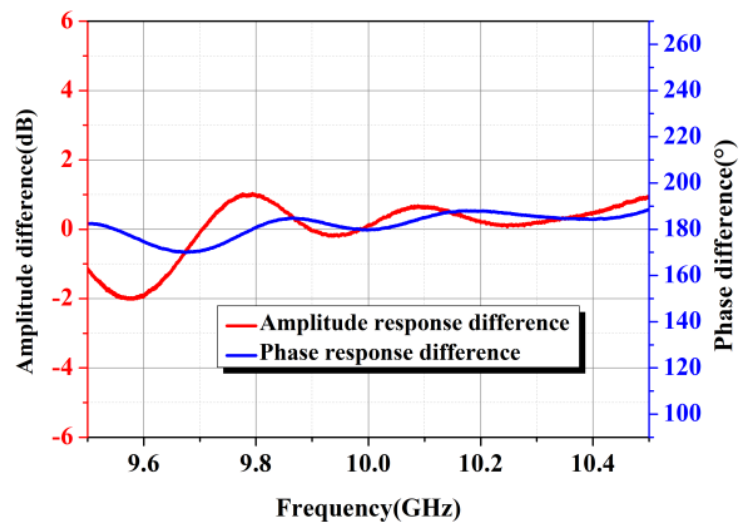

Figure 7. Amplitude and phase response difference between the lower and the upper paths.

\section{CONCLUSION}

The photonic RF SIC scheme based on phase modulation and optical single sideband filtering has been presented. It avoids the bias voltage control as needed for the conventional intensity modulation scheme. The factors influencing performance of the photonic RF SIC system including the time delay and amplitude deviations are analyzed. The 
cancellation depth of $34.5 \mathrm{~dB}$ is measured for $10 \mathrm{GHz}$ signal with bandwidth of $50 \mathrm{MHz}$. The cancellation depth over different bandwidths was investigated according to the measured amplitude and phase difference between the lower and the upper paths in the photonic RF SIC system.

\section{Acknowledgements}

This work was supported in part by National Science Foundation of China under grant 61875028,61704017 and 61605023, National Pre-Research Foundation of China under grant 6140450010305 and 61404130403, Dalian Science and Technology Innovation Foundation under grant 2018J11CY006, and Fundamental Research Funds for the Central Universities under grant DUT18ZD106, DUT18GF102, and DUT18LAB20.

\section{REFERENCES}

[1] A. Sabharwal, P. Schniter, D. Guo, D. W. Bliss, S. Rangarajan, and R. Wichman, "In-band full-duplex wireless: challenges and opportunities,” IEEE J. Sel. Areas Comm, vol. 32, no. 9, pp.1637 - 1652, Sept. 2014.

[2] S. K. Sharma, T. E. Bogale, L. B. Le, S. Chatzinotas, X. Wang and B. Ottersten, "Dynamic spectrum sharing in 5G wireless networks with full-duplex technology: recent advances and research challenges," IEEE Communications Surveys \& Tutorials, vol. 20, no. 1, pp. 674-707, First quarter 2018.

[3] Q. Zhou, H. Feng, G. Scott, and M. P. Fok, "Wideband co-site interference cancellation based on hybrid electrical and optical techniques,” Optics Letters, vol. 39, no. 22, pp. 6537 - 6540, Nov. 2014.

[4] G. Boudreau, J. Panicker, N. Guo, R. Chang, N. Wang, and S. Vrzic, "Interference coordination and cancellation for 4G networks,” IEEE Communications Magazine, Vol. 47, No. 4, pp. 74 - 81, Apr. 2009.

[5] S. Hong, J. Brand, J. I. Choi, M. Jain, J. Mehlman, S. Katti, and P. Levis, "Applications of self-interference cancellation in 5G and beyond,” IEEE Communications Magazine, vol. 52, no. 2, pp. 114 - 121, Feb. 2014.

[6] J. Suarez, K. Kravtsov, and P. R. Prucnal, "Incoherent method of optical interference cancellation for radiofrequency communications,” IEEE J. Quantum Electron. Vol. 5, No.4, pp. 402 - 408, April 2009.

[7] J. J. Sun, M. P. Chang and P. R. Prucnal, "Demonstration of Over-the-Air RF Self-Interference Cancellation Using an Optical System,” IEEE Photonics Technology Letters, Vol. 29, No. 4, pp. 397-400, Feb. 2017.

[8] Y. Chen and S. Pan, "Simultaneous wideband radio-frequency self-interference cancellation and frequency downconversion for in-band full-duplex radio-over-fiber systems," Optics Letters, vol. 43, no. 13, pp. 31243127, July 2018.

[9] W. Zhou, P. Xiang, Z. Niu, M. Wang and S. Pan, “Wideband Optical Multipath Interference Cancellation Based on a Dispersive Element," in IEEE Photonics Technology Letters, vol. 28, no. 8, pp. 849-851, 15 April15, 2016.

[10]X. Han, B. Huo, Y. Shao, M. Zhao, "Optical RF self-interference cancellation by using an integrated dualparallel MZM,” IEEE Photon. J., vol. 9, no. 2, Apr. 2017.

[11]N. Shi, Q. Song, J. Tang, W. Li, N. Zhu, and M. Li, “A switchable self-interference cancellation system for dual-band IBFD system using a monolithic integrated DML array,” Optics Communications, vol. 447, no. 15 pp. 55-60, Sept. 2019.

[12] L. Huang, Y. Zhang, S. Xiao, L. Zheng, and W. Hu, "Real-time adaptive optical self-interference cancellation system for in-band full-duplex transmission," Optics Communications, vol. 437, no. 15, pp. 259-263, April 2019.

[13] M. P. Chang, E. C. Blow, J. J. Sun, M. Z. Lu and P. R. Prucnal, “Integrated Microwave Photonic Circuit for Self-Interference Cancellation,” in IEEE Transactions on Microwave Theory and Techniques, vol. 65, no. 11, pp. 4493-4501, Nov. 2017.

[14]M. P. Chang, E. C. Blow, M. Z. Lu, J. J. Sun and P. R. Prucnal, "RF Characterization of an Integrated Microwave Photonic Circuit for Self-Interference Cancellation,” in IEEE Transactions on Microwave Theory and Techniques, vol. 66, no. 1, pp. 596-605, Jan. 2018. 